\title{
The influence of anemia on one-year exacerbation rate of patients with COPD-PH
}

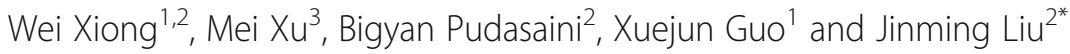

\begin{abstract}
Background: Anemia is prevalent not only in COPD but also in pulmonary hypertension. We postulated that anemia may have certain prognostic value in COPD concomitant with PH due to COPD (COPD-PH).

Methods: We performed a 12-month prospective investigation to follow up COPD patients with or without PH assessed by right heart catheterization. Eligible patients were enrolled, stratified into COPD-PH-anemia group $(n=40)$, COPD-PH group $(n=42)$, COPD-anemia group $(n=48)$, and COPD group $(n=50)$, and then followed up for 12 months.

Results: After the follow-up, for both of the actual variation value and variation rate, the increase of NT-pro BNP $(P<0.001$; $P=0.03)$ and CAT score $(P=0.001 ; 0.002)$, as well as the decrease of $\mathrm{PaO}_{2}(P=0.03 ; 0.086)$ and $P_{\text {eak }} \mathrm{VO}_{2}(P=0.021 ; 0.009)$ in COPD-PH-anemia group were highest among four groups. The cumulative one-year survival rates were similar among four groups $(P=0.434)$. The cumulative exacerbation-free rate was lowest in COPD-PH-anemia group among four groups $(P<0.001)$. Hemoglobin was an independent promoting factor for the probability of hospitalization due to exacerbation $\geqq 1 /$ year in patients with COPD-PH-anemia [HR 3.121(2.325-5.981); $P<0.001]$.

Conclusions: Anemia is a promoting factor for the worsening of exercise capacity, deterioration of hypoxemia, declining of life quality, and aggravation of exacerbations in patients with COPD-PH-anemia, by contrast with COPD-PH, COPDanemia, and COPD.
\end{abstract}

Keywords: COPD, Pulmonary hypertension, Anemia, Prognosis, Hemoglobin

\section{Background}

Chronic obstructive pulmonary disease (COPD) has become the third leading cause of death worldwide and is projected to be the disease with the seventh greatest burden worldwide in 2030. It is a major chronic cause of morbidity and mortality all over the world. Many patients suffer from this disease for many years, and die prematurely due to itself or its complications [1-3].

Pulmonary hypertension $(\mathrm{PH})$ is a pathophysiological disorder involving multiple clinical disciplines, which majorly include multiple cardiovascular and respiratory diseases [4]. It may develop in the advanced stage of COPD and is basically due to hypoxic vasoconstriction of pulmonary capillaries, eventually leading to structural changes which include intimal hyperplasia and the

\footnotetext{
* Correspondence: jinmingliu2013@126.com

Xuejun Guo and Jinming Liu are joint corresponding authors

${ }^{2}$ Department of Cardiopulmonary Circulation, Shanghai Pulmonary Hospital,

Tongji University School of Medicine, Shanghai, China

Full list of author information is available at the end of the article
}

consequent smooth muscle hypertrophy [5-7]. Once $\mathrm{PH}$ develops in patients with COPD, what may follow are the deteriorated exercise capacity, worsened hypoxemia and shortened survival [8-10].

In patients with COPD, systemic inflammatory mediators may contribute to skeletal muscular atrophy or cachexia, and initiate or aggravate anemia [11], meanwhile, anemia is common in patients with $\mathrm{PH}$ and may be associated with reduced exercise capacity, and with a higher mortality [12-16]. Therefore, since anemia had been evident to be prevalent in both COPD and $\mathrm{PH}$, we wondered how the prognostic role anemia was in $\mathrm{PH}$ due to COPD. For patients with COPD related PH, there are many shared prognostic factors between COPD and $\mathrm{PH}$, such as $\mathrm{DLcO}, 6 \mathrm{MWD}, \mathrm{PaO}_{2}, \mathrm{mMRC}$ score and peak $\mathrm{VO}_{2}$ which all decline worse than in either of $\mathrm{COPD}$ or $\mathrm{PH}$ alone providing the inter-related basis for the assessment of COPD-PH. To date, no existing studies have concerned this subject. Therefore, this study was

(c) The Author(s). 2018 Open Access This article is distributed under the terms of the Creative Commons Attribution 4.0 International License (http://creativecommons.org/licenses/by/4.0/), which permits unrestricted use, distribution, and 
designed to explore the potential prognostic value of anemia in PH due to COPD.

\section{Methods \\ Study design}

We performed a 12-month prospective study to investigate the role of anemia in COPD concomitant with $\mathrm{PH}$. All eligible patients were screened out according to inclusion and exclusion criteria and then stratified into COPD-PH-anemia group, COPD-PH group, COPDanemia group, and COPD group, to be followed up for 12 months. Variables encompassing routine blood test (RBT), COPD assessment test (CAT), pulmonary function test (PFT), cardiopulmonary exercise test (CPET), $6 \mathrm{~min}$ walk distance (6MWD), and arterial blood gas analysis (ABGA) were assessed at the baseline and the endpoint. Cumulative exacerbation counting and all-cause mortality were documented during the follow-up. All relevant variables were compared amongst the four groups after the finish of follow-up. During the enrollment of the patients, we equalized the variables of factors which might impact the prognosis to the maximum extent except for hemoglobin. Since our subjects were primarily patients with COPD, so we focused mainly on the equalization of GOLD stage, AE history, and co-morbidities. Meanwhile, we eliminated the difference of therapies by standardizing the treatment according to the guidelines. This protocol was approved by the institutional review board of Shanghai Pulmonary Hospital. Written informed consent was obtained from all patients.

\section{Study population}

All eligible patients were enrolled from a cohort of patients with COPD with or without $\mathrm{PH}$ assessed by right heart catheterization (RHC) between 2013 and 2016 of the department of cardiopulmonary circulation of Shanghai Pulmonary Hospital Tongji University, due to at least one of the following reasons: 1) episodes of RV failure or suspected $\mathrm{PH}$ by echocardiographic findings; 2) suspected $\mathrm{PAH}$ or $\mathrm{CTEPH}$; 3) candidates for lung transplantation or lung volume reduction.

Eligible patients were enrolled according to the inclusion criteria and the exclusion criteria. Inclusion criteria: 1) age $\geq 40$ yrs.; 2) a diagnosis of COPD at all stages/ groups, defined as an $\mathrm{FEV}_{1}$ : FVC ratio of less than 0.70 after bronchodilator use plus respiratory symptoms, a history of exposure to risk factors (e.g., smoking, air pollution, biomass combustion), or both, measured $20 \mathrm{~min}$ after the inhalation of $400 \mu \mathrm{g}$ of albuterol (Ventolin, Glaxo Wellcome) [11]; 3) a diagnosis with $\mathrm{PH}$ on the presence of mean pulmonary arterial pressure (mPAP) $\geqq 25 \mathrm{mmHg}$ and pulmonary artery wedge pressure (PAWP) $\leq 18 \mathrm{mmHg}$ in RHC or an exclusion of $\mathrm{PH}$ by $\mathrm{mPAP}<25 \mathrm{mmHg}$ in RHC [4]; 4) with or without a diagnosis of anemia defined as a hemoglobin concentration of $<13 \mathrm{~g} / \mathrm{dL}$ for males and $12 \mathrm{~g} / \mathrm{dL}^{-}$for females [17]. Exclusion criteria: 1) a diagnosis of other chronic pulmonary diseases including, asthma or asthma-COPD overlap (ACO), bronchiectasis, tuberculosis, obliterative bronchiolitis, diffuse panbronchiolitis, interstitial lung disease, or combined pulmonary fibrosis and emphysema; 2) a diagnosis of PH in Group 1, Group 2, Group 4, or Group 5 according to the classifications in 2015 ESC/ERS guidelines [4]; 3) patients with hematological diseases including secondary anemia such as anemia due to cancer or immunological diseases or hemorrhage, or receive regimens which affect hemoglobin except for anti-anemia therapy; 4) patients who lived on plateau all the time; 5) patients who were lost to follow-up or who did not comply with COPD-related or PH-related treatments.

\section{Assessments}

We performed the assessments encompassing several aspects which were exercise capacity, hypoxemia, life quality, acute exacerbation and all-cause mortality. The detailed variables we focused were hemoglobin, carboxyhemoglobin, methemoglobin in RBT, $\mathrm{PaO}_{2}$ in $\mathrm{ABGA}$, $\mathrm{FEV}_{1}$ of the predicted value in PFT, peak $\mathrm{VO}_{2}$ in CPET, CAT score, NT-pro BNP and 6MWD. All assessments were performed when patients were at their stable status. In case of patients happened to be in an exacerbated state at the moment of assessment, the evaluation would be postponed till patients recovered from exacerbations. Exacerbation was defined as an acute worsening of respiratory symptoms that result in additional therapy $[18,19]$. At the end of each month during the follow-up, study personnel determined the patients' status including exacerbations, hospitalizations due to exacerbations, and survival status in the previous month by telephone contact.

\section{Statistical analysis}

According to the prevalence of COPD (11.7\%), the anemia prevalence in COPD (12.3-23\%), and the prevalence of $\mathrm{PH}$ in COPD (50-90\%), to ensure the 95\% confidential interval, we estimated we at least needed to measure in total of 159 cases of COPD patients, in which at least 82 cases of COPD-anemia in total, 75 cases of COPD-PH in total, and at least 36 cases of COPD-PH-anemia.

Measurement data was presented as mean \pm standard deviation or median with interquartile range according to their distribution. Categorical data was presented as frequencies and percentages. Exacerbation-free rates and survival rates at different time-points were estimated by means of Kaplan-Meier method, and any differences between groups were evaluated with a stratified log-rank test. The multiple testing among all groups was conducted by using ANOVA with Bonferroni correction. 
The change of patients' variables between the baseline and the study completion was calculated: change $=($ variable at completion- variable at baseline); the change rate was calculated: change rate $=$ (variable at completion-variable at baseline)/variable at baseline. Cox regression analysis was performed to assess the correlation between variables and the probability of hospitalizations due to exacerbations $\geqq$ 1 time per year. A $p$-value $<0.05$ was defined as being of statistical significance.

\section{Results}

\section{Demographics and characteristics of the patients}

This investigation was launched in January, 2016, and finished in December, 2017, following the finish of follow-up of the last enrolled patient. After the exclusion of 10 cases with at least one of the following diagnoses of asthma, bronchiectasis, tuberculosis, obliterative bronchiolitis, diffuse panbronchiolitis, interstitial lung disease, or combined pulmonary fibrosis and emphysema, 6 cases with a diagnosis of PH in Group 1, Group 2 , Group 4, or Group 5, and 3 cases with hematological diseases, or receive regimens which affect hemoglobin except for anti-anemia therapy, amongst 207 cases, finally, a total of 188 eligible patients were screened out to access to the follow-up program. Then after the loss of 8 cases to the follow-up, in the end, 180 cases entered into the final full analysis set. Throughout all of them, the cases in COPD-PH-anemia group, COPD-PH group, COPD-anemia group, and COPD group were 40, 42, 48, and 50 , respectively. The overall mean age and male/female sex ratio of all eligible patients were 66.1 years and
$131 / 49$, respectively. No statistical difference was found among four groups in regard to age, sex ratio, smoking history, AE history, $\mathrm{FEV}_{1}$, GOLD stages, and COPD groups $(P>0.05$ for all comparisons), except for BMI $(P$ $=0.025), \mathrm{mPAP}(P=0.016), 6 \mathrm{MWD}(P=0.003)$, NT-pro BNP $(P<0.001), \mathrm{PaO}_{2}(P=0.006)$, peak $\mathrm{VO}_{2}(P=0.018)$ and hemoglobin $(P=0.036)$ at the baseline. By means of a routine blood test, among 88 cases with anemia, 45 cases were identified to be normocytic anemia, 33 cases were microcytic anemia, and 10 cases were macrocytic anemia. Demographics and characteristics of the patients at the baseline were summarized in Table 1. LTOT was prescribed according to patients' indication before and during the study period. No statistical difference regarding LTOT was found among four groups $(P=0.085)$.

\section{Comparison of variation of variables between the baseline and the endpoint among four groups}

The results demonstrated that no statistical difference were found regarding the $\mathrm{FEV}_{1}$ among four groups in both aspects of actual variation value and variation rate $(P=0.057 ; 0.062)$. Except the variation rates of $\mathrm{PaO}_{2}$ were similar among four groups $(P=0.086)$, no matter regarding actual variation value or variation rate, the increase of NT-pro BNP $(P<0.001 ; P=0.03)$ and CAT score $(P=$ $0.001 ; 0.002)$ in COPD-PH-anemia group were significantly highest among four groups, whereas the decrease of $\mathrm{PaO}_{2}(P=0.03 ; 0.086)$ and Peak $\mathrm{VO}_{2}(P=0.021 ; 0.009)$ in COPD-PH-anemia group were significantly highest among four groups (Table 2).

Table 1 Demographics and characteristics of all the patients at the baseline

\begin{tabular}{|c|c|c|c|c|c|}
\hline Variables & COPD-PH-anemia $(n=40)$ & COPD-PH $(n=42)$ & COPD-anemia $(n=48)$ & $\operatorname{COPD}(n=50)$ & $P$ value \\
\hline Age-yrs & $65.9 \pm 5.3$ & $68.7 \pm 8.1$ & $62.6 \pm 6.7$ & $67.2 \pm 7.5$ & 0.587 \\
\hline Sex (M/F)-\% & $75.0 / 25.0$ & $71.4 / 28.6$ & $68.8 / 31.2$ & $76.0 / 24.0$ & 0.661 \\
\hline $\mathrm{BMI}-\mathrm{kg} / \mathrm{m}^{2}$ & $18.5 \pm 5.8$ & $21.6 \pm 6.2$ & $22.4 \pm 4.7$ & $25.1 \pm 7.1$ & 0.025 \\
\hline Smoking history (Y/N)-\% & $90.0 / 10.0$ & $88.1 / 11.9$ & $85.4 / 14.6$ & $86.0 / 14.0$ & 0.549 \\
\hline $\mathrm{FEV}_{1}$ of predicted value- $\%$ & $40.7 \pm 25.9$ & $43.8 \pm 19.6$ & $47.7 \pm 22.6$ & $42.3 \pm 17.8$ & 0.383 \\
\hline AE history- no. & $2.8 \pm 1.5$ & $2.5 \pm 2.3$ & $2.6 \pm 1.7$ & $2.2 \pm 1.8$ & 0.446 \\
\hline GOLD (I/II/III/IV)-\% & $0 / 7.5 / 47.5 / 45.0$ & $0 / 11.9 / 45.2 / 42.9$ & 0/8.3/41.7/50.0 & 0/10.0/43.1/46.9 & 0.125 \\
\hline Group (A/B/C/D)-\% & $0 / 12.5 / 40.0 / 47.5$ & 0/14.3/47.6/38.1 & 0/14.6/43.8/41.6 & 0/16.0/40.0/44.0 & 0.374 \\
\hline mPAP-mmHg & $33.8 \pm 18.5$ & $30.2 \pm 22.6$ & $20.6 \pm 15.3$ & $18.9 \pm 25.4$ & 0.016 \\
\hline CAT score-points & $23.5 \pm 10.2$ & $16.9 \pm 13.8$ & $22.2 \pm 8.6$ & $18.7 \pm 15.1$ & 0.186 \\
\hline 6MWD-m & $298.9 \pm 183.4$ & $353.6 \pm 164.7$ & $382.1 \pm 173.7$ & $452.3 \pm 152.2$ & 0.003 \\
\hline NT-pro BNP-ng/L & $1608.2 \pm 679.8$ & $1344.9 \pm 852.4$ & $556.7 \pm 391.1$ & $374.6 \pm 421.0$ & $<0.001$ \\
\hline $\mathrm{PaO}_{2}-\mathrm{mmHg}$ & $45.3 \pm 13.8$ & $50.7 \pm 18.1$ & $55.6 \pm 16.4$ & $65.7 \pm 17.2$ & 0.006 \\
\hline Peak $\mathrm{VO}_{2}-\mathrm{ml} / \mathrm{min} / \mathrm{kg}$ & $13.9 \pm 5.7$ & $15.2 \pm 7.3$ & $18.5 \pm 6.2$ & $22.2 \pm 8.1$ & 0.018 \\
\hline Hemoglobin-g/dL ${ }^{-1}$ & $9.4 \pm 5.5$ & $14.2 \pm 6.7$ & $10.7 \pm 8.3$ & $13.8 \pm 4.9$ & 0.036 \\
\hline
\end{tabular}

Note: COPD chronic obstructive pulmonary disease, $P H$ pulmonary hypertension, $B M I$ body mass index, $F E V_{1}$ forced expiatory volume in $1 \mathrm{~s}, A E$ acute exacerbation, GOLD global Initiative for Chronic Obstructive lung Disease, mPAP mean pulmonary arterial pressure, CAT COPD assessment test, 6MWD 6-min walking distance, NT-proBNP N-terminal pro-brain natriuretic peptide, $\mathrm{PaO}_{2}$ arterial blood oxygen tension, $P$ eak $\mathrm{VO}_{2}$ peak oxygen consumption 
Table 2 Comparison of the change and changing rate of patients' variables between the baseline and the endpoint among four groups

\begin{tabular}{llllll}
\hline Variables & COPD-PH-anemia $(n=40)$ & COPD-PH $(n=42)$ & COPD-anemia $(n=48)$ & COPD $(n=50)$ & $P$ value \\
\hline FEV $_{1}$-\% (\%) & $-8.8 \pm 3.6(-10.1 \pm 5.3)$ & $-8.5 \pm 6.4(-10.6 \pm 4.6)$ & $-7.2 \pm 5.1(-8.3 \pm 7.2)$ & $-7.6 \pm 4.2(-9.1 \pm 4.8)$ & $0.057(0.062)$ \\
CAT score-points (\%) & $12.6 \pm 5.8(23.7 \pm 15.1)$ & $6.5 \pm 3.7(19.2 \pm 12.4)$ & $6.6 \pm 4.0(22.3 \pm 10.6)$ & $4.7 \pm 3.2(20.1 \pm 8.8)$ & $0.001(0.002)$ \\
6MWD-m (\%) & $-59.5 \pm 45.6(-32.2 \pm 18.8)$ & $-34.3 \pm 41.2(-26.5 \pm 14.3)$ & $-28.4 \pm 40.1(-20.5 \pm 16.2)$ & $-19.7 \pm 38.3(-16.5 \pm 12.3)$ & $0.007(0.01)$ \\
NT-pro BNP- ng/L (\%) & $597.1 \pm 154.4(31.3 \pm 20.4)$ & $466.8 \pm 191.0(25.5 \pm 22.3)$ & $125.7 \pm 112.1(19.5 \pm 17.2)$ & $133.6 \pm 108.5(15.2 \pm 13.3)$ & $<0.001(0.03)$ \\
$\mathrm{PaO}_{2}-\mathrm{mmHg}_{\text {(\%) }}$ & $-10.7 \pm 5.8(-10.9 \pm 8.6)$ & $-7.6 \pm 5.3(-11.7 \pm 7.0)$ & $-6.6 \pm 5.4(-8.8 \pm 7.5)$ & $-4.9 \pm 4.5(-7.9 \pm 7.2)$ & $0.03(0.086)$ \\
Peak VO $\mathrm{V}_{2}-\mathrm{ml} / \mathrm{min} / \mathrm{kg}(\%)$ & $-3.5 \pm 1.6(-32.4 \pm 10.3)$ & $-2.8 \pm 1.9(-25.5 \pm 13.6)$ & $-2.4 \pm 2.1(-17.7 \pm 8.8)$ & $-1.8 \pm 1.4(-10.3 \pm 11.7)$ & $0.021(0.009)$
\end{tabular}

Note: $F E V_{1}$ forced expiatory volume in $1 \mathrm{~s}, C A T$ COPD assessment test, $6 M W D$ 6-min walking distance, NT-proBNP N-terminal pro-brain natriuretic peptide, PaO ${ }_{2}$ arterial blood oxygen tension, Peak $\mathrm{VO}_{2}$ peak oxygen consumption

Comparison of cumulative overall survival, exacerbationfree rate amongst four groups

At the end of the follow-up, the cumulative overall mortality were 19 cases, in which 7cases were in COPD$\mathrm{PH}$-anemia group, 5 cases were in COPD-PH group, 4 cases were in COPD-anemia group, and 3cases were in $\operatorname{COPD} \operatorname{group}(P=0.096)$. Among all the deceased, 10 patients died of respiratory failure, 7 patients died of heart failure, 2 cases died of sudden death. In a Kaplan-Meier analysis, the results demonstrated that the cumulative one-year survival rates were similar amongst COPD$\mathrm{PH}$-anemia group, COPD-PH group, COPD-anemia group, and COPD group $(P=0.434)$ (Fig. 1.) Throughout the whole process of follow-up, the mean annual exacerbations or hospitalizations counting per patient were 3.5 and 1.8 times in COPD-PH-anemia group, 2.6 and 1.7 times in COPD-PH group, 2.4 and 1.3 times in COPD-anemia group, as well as 1.8 and 0.8 times in COPD group, respectively $(P=0.005 ; P=0.018)$. At the end of the follow-up, the cases with at least one exacerbation or one hospitalization were 118(65.6\%) and 66 (36.7\%) cases, respectively. The prevalence of exacerbations or hospitalizations were $35(87.5 \%)$ and $16(40.0 \%)$ in COPD-PH-anemia group, 28(66.7\%) and $15(35.7 \%)$ in COPD-PH group, 30(62.5\%) and 12(25.0\%) in COPDanemia group, as well as $25(50 \%)$ and 10(20.0\%) in COPD group $\quad[P=0.033(P<0.001) ; P=0.065(P=0.005)]$. In a Kaplan-Meier analysis, the results demonstrated that the cumulative exacerbation-free proportion was lowest in COPD-PH-anemia group, and highest in COPD group, whereas no statistical difference was found between COPD-PH group and COPD-anemia $\operatorname{group}(P<0.001)$ (Fig. 2.).

\section{Correlation between risk factors and exacerbations in each group by multivariate regression analysis}

After an univariate analysis between risk factors and the development of hospitalizations due to exacerbations $\geqq 1$ / year, then adjusting for age, sex, smoking history and BMI, a multivariate analysis demonstrated that, for patients with COPD-PH-anemia, along with per decrease of $1 \mathrm{~g} / \mathrm{dL}^{-1}$ of hemoglobin, the hazard ratio of hospitalizations $\geqq 1 /$ year was 3.121, being similar with some variables such as $\mathrm{AE}$ history and COPD groups. Also in a multivariate regression analysis between dyshemoglobins which were carboxyhemoglobin as well as methemoglobin and the risk for hospitalizations $\geqq 1 /$ year, the results showed that only carboxyhemoglobin was positively correlated with the development of hospitalizations $\geqq 1 /$ year especially in COPD-PH-anemia group (Table 3.)

\section{Discussion}

In consideration of anemia may have certain prognostic value in patients with pulmonary hypertension due to COPD, whereas little of them was known quoad hoc, thus we performed this study. In this study, we found that, among COPD-PH-anemia group, COPD-PH group, COPD-anemia group, and COPD group, the patients in COPD-PH-anemia group had the most deterioration in exercise capacity, hypoxemia, life quality, and highest risk of acute exacerbations, except for the similar overall survival rates among all groups, in a 12-month interval.

To our best knowledge, no existing comparable study is eligible to be the contrast with this study, therefore, what we can discuss hereby is this investigation exclusively. Since PH is also a concomitant co-morbidity just like anemia, we primarily regarded the subjects as COPD patients, then as $\mathrm{PH}$ or not. In order to present the the impact of anemia on COPD-PH to the maximum extent, we set up not only COPD-PH, but also COPD-anemia and COPD as control. Besides the information of impact of anemia on COPD-PH, we could also obtain the information regarding the different impact of anemia on COPD-PH and COPD, respectively, by contrast with sole COPD. It cannot be denied that secondary polycythemia is a common phenomenon in patients with COPD just like anemia, in other words, the two pathophysiologic processes may potentially happen in patients with COPD simultaneously, especially in early stage of COPD. Therefore, since the basic hemoglobin level of COPD may be higher than that of normal person, we adopted the diagnostic criteria of WHO for anemia which are 


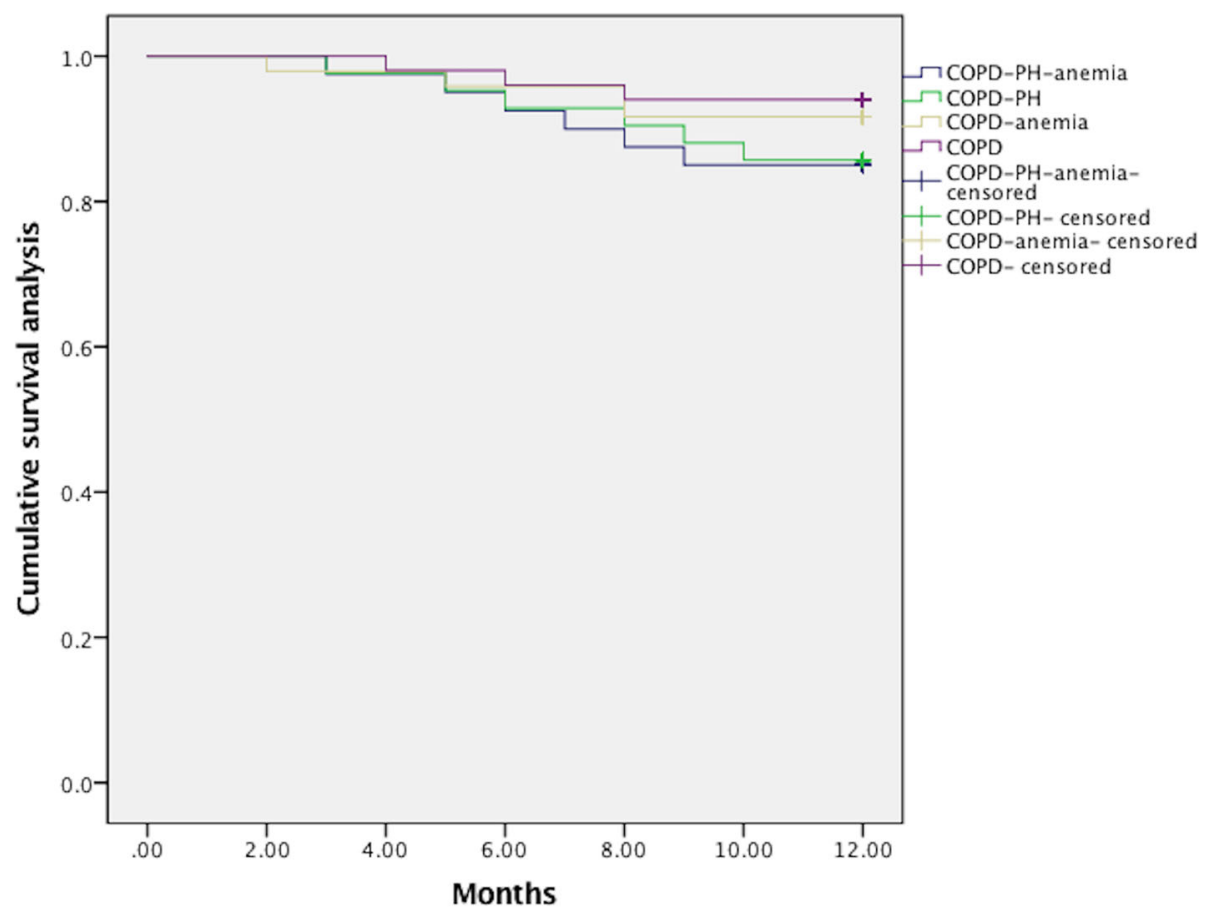

Fig. 1 Comparison of cumulative one-year overall survival rate among COPD-PH-anemia group, COPD-PH group, COPD-anemia group, and COPD group $(P=0.434)$

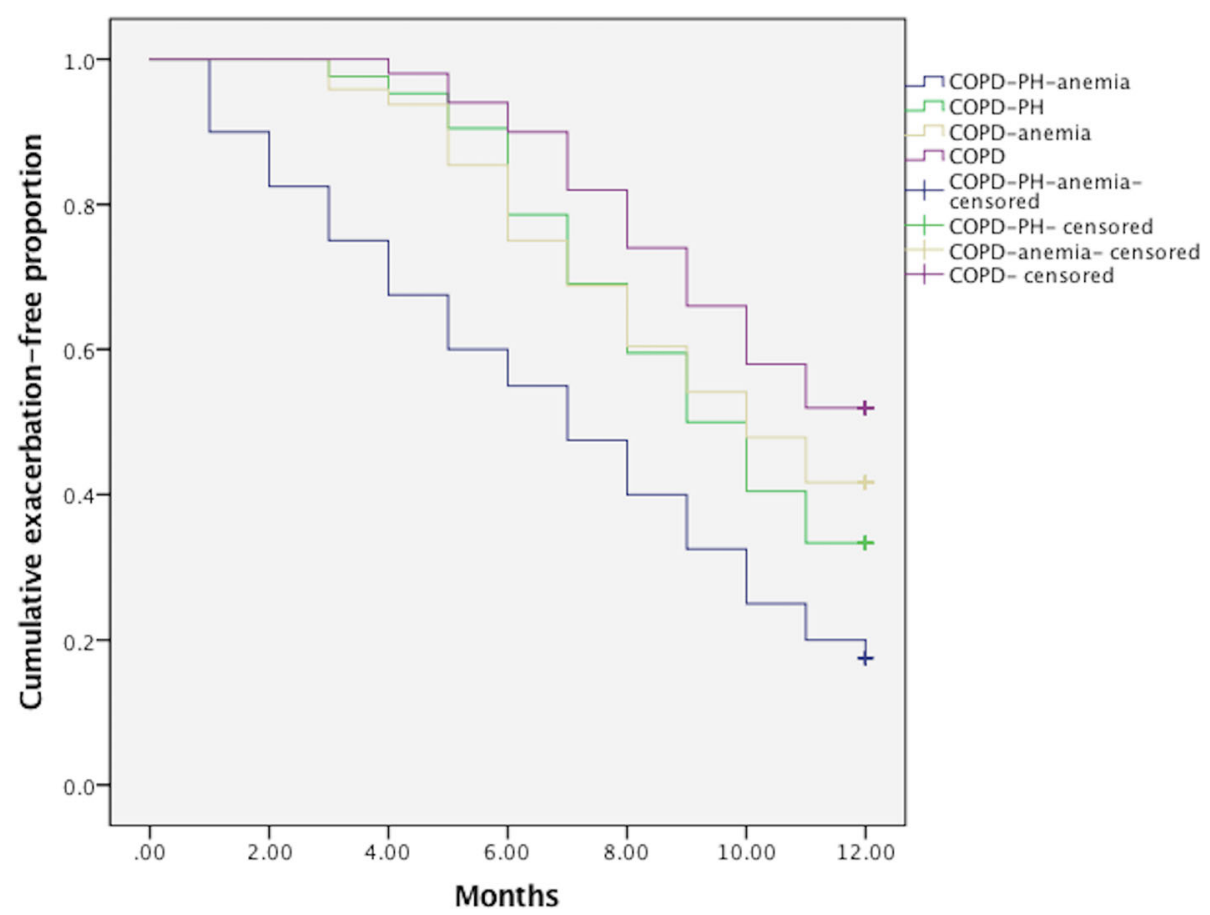

Fig. 2 Comparison of cumulative one-year exacerbation-free rate among COPD-PH-anemia group, COPD-PH group, COPD-anemia group, and COPD group $(P<0.001)$ 
Table 3 Correlation between risk factors and the probability of hospitalizations $\geqq 1 /$ year in each group by multivariate regression analysis

\begin{tabular}{|c|c|c|c|c|}
\hline Variables & COPD-PH-anemia $(n=40)$ & COPD-PH $(n=42)$ & COPD-anemia $(n=48)$ & $\operatorname{COPD}(n=50)$ \\
\hline & HR $(95 \% C l), P$ value & HR $(95 \% \mathrm{Cl}), P$ value & HR $(95 \% \mathrm{Cl}), P$ value & HR $(95 \% \mathrm{Cl}), P$ value \\
\hline $\mathrm{FEV}_{1}$-per decrease of $10 \%$ & $2.565(1.225-3.764) 0.003$ & $2.439(1.219-3.664) 0.002$ & $2.108(1.321-4.214) 0.005$ & 1.884(0.871-3.265) 0.003 \\
\hline AE history-per increase of 1 time & $3.338(1.532-4.698)<0.001$ & $3.157(1.339-5.310)<0.001$ & $2.541(1.210-4.311) 0.001$ & $2.226(1.722-3.827) 0.001$ \\
\hline GOLD-per progression of 1 stage & $2.765(1.555-3.827) 0.008$ & $2.672(1.246-3.981) 0.005$ & $2.519(1.433-3.646) 0.001$ & $1.987(0.592-3.218) 0.037$ \\
\hline Group-per progression of 1 level & $3.102(1.426-3.223)<0.001$ & $2.453(1.002-3.528) 0.002$ & $2.313(1.038-3.297) 0.004$ & $2.124(0.762-3.281) 0.014$ \\
\hline $\mathrm{PaO}_{2}$-per decrease of $10 \mathrm{mmHg}$ & $2.384(1.542-3.456) 0.02$ & $2.176(1.256-3.642) 0.006$ & $2.987(1.777-3.562) 0.007$ & $1.733(0.888-3.213) 0.028$ \\
\hline $\begin{array}{l}\text { Peak } \mathrm{VO}_{2} \text {-per decrease of } \\
10 \mathrm{ml} / \mathrm{min} / \mathrm{kg}\end{array}$ & $2.182(1.214-3.628) 0.003$ & $2.815(1.118-3.823) 0.033$ & $2.143(1.143-3.427) 0.004$ & $2.054(1.076-3.665) 0.029$ \\
\hline 6MWD-per decrease of $100 \mathrm{~m}$ & $1.676(0.662-3.186) 0.036$ & $1.501(0.855-3.222) 0.044$ & 1.453(0.337-3.212)0.038 & $1.222(0.443-3.251) 0.057$ \\
\hline $\begin{array}{l}\text { Hemoglobin -per decrease } \\
\text { of } 1 \mathrm{~g} / \mathrm{dL}^{-1}\end{array}$ & $3.121(2.325-5.981)<0.001$ & 1 (reference) & $2.756(1.985-3.784)<0.001$ & 1 (reference) \\
\hline $\begin{array}{l}\text { Carboxyhemoglobin-per } \\
\text { increase of } 0.1 \mathrm{~g} / \mathrm{dL}^{-1}\end{array}$ & $2.838(1.698-5.210) 0.001$ & $2.663(1.520-3.228) 0.001$ & $2.437(1.265-3.884) 0.001$ & $1.688(1.104-3.651) 0.002$ \\
\hline
\end{tabular}

$<13 \mathrm{~g} / \mathrm{dL}$ for males and $<12 \mathrm{~g} / \mathrm{dL}$ for females, respectively, instead of the criteria of anemia in China which are $<12 \mathrm{~g} / \mathrm{l}$ for male and $<11 \mathrm{~g} / \mathrm{l}$ for female, respectively, to eliminate the potential confounding of secondary polycythemia.

To start with, except for hemoglobin which was predetermined to be different among four groups, the demographics showed that no statistical difference was found in regard to age, sex ratio, smoking history, AE history, $\mathrm{FEV}_{1}$, GOLD stages, and COPD groups, suggesting the homogeneity was considerable at least from the perspective of COPD, among all eligible patients at the baseline. Nevertheless, some variables such as mPAP, 6MWD, NT-pro BNP, $\mathrm{PaO}_{2}$, and peak $\mathrm{VO}_{2}$ were heterogenous among all eligible patients at the baseline partially attributable to the role of $\mathrm{PH}$. Interestingly, the BMI in COPD-PH-anemia group was lowest among four groups suggesting anemia may interrelate with nutritional status. It is noteworthy that the cause of anemia was majorly due to normocytic type which conformed to the characteristics of COPD [11]. As for microcytic type being the second major cause, we believe it is related to $\mathrm{PH}$ [12-16].

After the follow-up, the results showed no dramatic variation regarding the $\mathrm{FEV}_{1}$ which is a COPD-related variable concerning airflow limitation, whereas the variations of NT-pro BNP, CAT score, $\mathrm{PaO}_{2}$ and Peak $\mathrm{VO}_{2}$ were significant among four groups in which the COPD-PH-anemia group had the worst deterioration. This indicated that, anemia impacted more seriously on patients with COPD-PH than on mere COPD, encompassing the perspectives of life quality, ventricular dysfunction, and hypoxemia especially whilst exercise, except for airflow limitation. On account of the impairment of oxygen-transporting function in anemia, patients with COPD-PH-anemia are naturally more liable to develop ingravescent fatigue, heart failure and hypoxemia rather than airflow limitation, by contrast with either COPD-PH or COPD.

The next comparison of cumulative overall survival showed no difference of cumulative one-year survival rates among four groups. This could be interpreted as that anemia makes no difference on the survival of patients with COPD-PH or COPD for at least 1 year. By contrast, in the study of Pernille et al., anemia could be used to predict mortality. In view of Pernille's study was a five-year retrospective review, while ours was a one-year prospective investigation, the investigating period in this study may be too limited to uncover the difference of mortality among different groups [20].

In the study of Pernille, et al., low level of hemoglobin are frequent in COPD patients with acute exacerbations [20]. In our study, the comparison of exacerbations demonstrated that COPD-PH-anemia group had the most mean annual exacerbations or hospitalizations counting, the highest prevalent rate of exacerbations or hospitalizations, and lowest cumulative exacerbation-free rate among patients among four groups. It means that, by contrast with simple COPD, anemic COPD, or simple COPD-PH, COPD-PH-anemia has the highest risk for developing an exacerbation. It is believed that, by deteriorating life quality, ventricular dysfunction, and hypoxemia, anemia contributes to the aggravation of exacerbations.

The last correlation analysis between risk factors and hospitalizations showed that, being similar with some exacerbation-related classical predictors in COPD such as AE history and COPD groups [11], hemoglobin was an independently contributing factor for the probability 
of hospitalizations $\geqq 1 /$ year in COPD patients especially patients with COPD-PH-anemia. Decremental hemoglobin is a promoting factor for the incremental exacerbations or hospitalizations. By the way, we also performed a correlation analysis between some dyshemoglobins which were carboxyhemoglobin as well as methemoglobin and hospitalizations. The results demonstrated that carboxyhemoglobin was positively correlated with the development of hospitalizations $\geqq 1 /$ year in all four groups especially in COPD-PH-anemia group rather than methemoglobin. Likewise, in the study of Yasuda et al., the carboxyhemoglobin level at exacerbations were significantly higher than those at stable stage, the increased arterial carboxyhemoglobin was correlated to the severity of COPD resulting from systemic inflammation and reactive oxygen species [21].

Some systematic inflammatory diseases such as connective tissue disease are frequently concomitant with anemia of chronic disease through the mechanism of the production of inflammatory mediators damaging the generation of erythrocytes. Likewise, COPD which is one of systematic inflammatory diseases is generally concomitant with the elevation of IL-1, IL-6 and TNF-a level in circulation inducing the development of anemia [22]. Some studies demonstrated that anemia was closely related to $\mathrm{C}$ reactive protein which is an inflammatory biomarker [23, 24]. Besides, inflammatory mediators may also result in skeletal muscular atrophy and cachexia further deteriorating anemia [11]. On the other hand, patients with pulmonary hypertension commonly develop right ventricular dysfunction in which $15 \%$ are concomitant with anemia [25-28]. Its mechanism is due to the release of inflammatory mediators whilst heart failure, the activation of renin-angiotensin system [28]. All these may explain the impressive prevalence of anemia in COPD-PH.

The clinical implications of this study are considered to be the following: first, the results of our study may urge clinicians to be aware of the serious prevalence of anemia in COPD patients concomitant with $\mathrm{PH}$; second, clinician could be vigilant about the severely adverse impact of anemia on the prognosis of COPD-PH in order to inform patients' family members timely and take action in advance; third, under some circumstances in which a dilemma exists in the assessment of prognosis, anemia could be an eligible weight which can be taken into account.

The strength of this study consisted in: first, the eligible patients being studied all underwent RHC which is the only gold standard for the diagnosis of $\mathrm{PH}$ to date, to ascertain wether they had $\mathrm{PH}$ or not, ensuring the eligibility of PH-negative COPD controls; second, we compared the longitudinal variation and variation rate between the baseline and the endpoint instead of comparing the variables at the endpoint, to reflect the time-dependent impact that anemia would result in. Nevertheless, several limitations existed in this study. First, the sample size was not very large due to the nature of prospective investigation. A large-scale study is warranted in the future. Second, obviously we have no comments to make on the potential difference of overall survival amongst different groups beyond one-year follow-up which might be too short to show the discrepancy. The last but not least, in view of the patients being reviewed in this study were all Chinese patients, the results of this study may not be applicable for other races.

\section{Conclusions}

In summary, in this study, we may draw a conclusion that anemia is a promoting factor for worse deterioration of exercise capacity, deterioration of hypoxemia, declining of life quality, as well as aggravation of exacerbations or hospitalizations in patients with COPD-PHanemia, by contrast with patients with COPD-PH, COPD-anemia, or COPD.

\section{Abbreviations \\ 6MWD: 6 min walk distance; ABGA: Arterial blood gas analysis; AE: Acute exacerbation; BMl: Body mass index; CAT: COPD assessment test; COPD: Chronic obstructive pulmonary disease; CPET: Cardiopulmonary exercise test; CTEPH: Chronic thromboembolic pulmonary hypertension; $\mathrm{FEV}_{1}$ : Forced expiatory volume in $1 \mathrm{~s}$; GOLD: Global Initiative for Chronic Obstructive lung Disease; mPAP: Mean pulmonary arterial pressure; NT- proBNP: N-terminal pro-brain natriuretic peptide; PAH: Pulmonary arterial hypertension; $\mathrm{PaO}_{2}$ : Arterial blood oxygen tension; PAWP: Pulmonary artery wedge pressure; Peak $\mathrm{VO}_{2}$ : Peak oxygen consumption; PFT: Pulmonary function test; ${ }_{i}$ PH: Pulmonary hypertension; RBT: Routine blood test; RHC: Right heart catheterization; RV: Right ventricular}

\section{Acknowledgements}

We sincerely thank Dr. Lan Wang, Dr. Jian Guo, Dr. Sugang Gong, Dr. Jing He, Dr. Qinhua Zhao, Dr. Rong Jiang, Dr. Cijun Luo, Dr. Hongling Qiu, Dr. Wenhui Wu, Dr. Minqi Liu, Dr. Tianxiang Chen, Dr. Xingxing Sun, and Dr. Chuanyu Wang of Department of Cardiopulmonary Circulation, Shanghai Pulmonary Hospital, Tongji University School of Medicine, Shanghai, China, for their assistance in this study.

\section{Funding}

This work was supported by the following funds: The Program of Shanghai Natural Science Foundation (16ZR1429000); the Program of Development Center for Medical Science and Technology, National Health and Family Planning Commission of the People's Republic of China (ZX-01-C2016144)

Availability of data and materials

Please contact Wei Xiong for data requests.

\section{Authors' contributions}

WX conceived of the study, and participated in its design, performance, statistics, coordination, drafting and revising of the manuscript. MX conceived of the study, and participated in its design, statistics, coordination, drafting and revising of the manuscript. XJG conceived of the study, and participated in its design, statistics, coordination, and revising of the manuscript. BP participated in its design, statistics, performance, coordination, drafting and revising of the manuscript. JML conceived of the study, and participated in its design, coordination and revising of the manuscript. All authors read and approved the final manuscript.

Ethics approval and consent to participate

This protocol was approved by the institutional review board of Shanghai Pulmonary Hospital. Written informed consent was obtained from all patients. 


\section{Consent for publication}

Not applicable

\section{Competing interests}

The authors declare that they have no competing interests.

\section{Publisher's Note}

Springer Nature remains neutral with regard to jurisdictional claims in published maps and institutional affiliations.

\section{Author details}

'Department of Respiratory Medicine, Xinhua Hospital, Shanghai Jiaotong University School of Medicine, No. 1665, Kongjiang Road, Yangpu District, Shanghai 200092, People's Republic of China. ${ }^{2}$ Department of Cardiopulmonary Circulation, Shanghai Pulmonary Hospital, Tongji University School of Medicine, Shanghai, China. ${ }^{3}$ Department of Pediatrics, Dinghai Community Health Service Center, Tongji University School of Medicine, Shanghai, China;Department of Pediatrics, Kongjiang Hospital, Yangpu District, Shanghai, China.

Received: 9 April 2018 Accepted: 19 July 2018

Published online: 23 August 2018

\section{References}

1. Lozano R, Naghavi M, Foreman K, et al. Global and regional mortality from 235 causes of death for 20 age groups in 1990 and 2010: a systematic analysis for the global burden of disease study 2010. Lancet. 2012;380:2095-128.

2. Yang G, Wang Y, Zeng Y, et al. Rapid health transition in China, 1990-2010: findings from the global burden of disease study 2010. Lancet. 2013;381: 1987-2015.

3. Mathers $C D$, Loncar D. Projections of global mortality and burden of disease from 2002 to 2030. PLoS Med. 2006;3(11):e442.

4. Galiè N, Humbert M, Vachiery J-L, et al. 2015 ESC/ERS guidelines for the diagnosis and treatment of pulmonary hypertension. Eur Respir J. 2015;46:879-82.

5. Sakao S, Voelkel NF, Tatsumi K. The vascular bed in COPD: pulmonary hypertension and pulmonary vascular alterations. Eur Respir Rev. 2014 23(133):350-5.

6. Peinado VI, Pizarro S, Barbera JA. Pulmonary vascular involvement in COPD Chest. 2008;134(4):808-14.

7. Wells JM, Washko GR, Han MK, et al. Pulmonary arterial enlargement and acute exacerbations of COPD. N Engl J Med. 2012;367(10):913-21.

8. Oswald-Mammosser M, Weitzenblum E, Quoix E, et al. Prognostic factors in COPD patients receiving long-term oxygen therapy. Importance of pulmonary artery pressure. Chest. 1995;107:1193-8.

9. Kessler R, Faller M, Weitzenblum E, et al. "Natural history" of pulmonary hypertension in a series of 131 patients with chronic obstructive lung disease. Am J Respir Crit Care Med. 2001;164:219-24.

10. Lettieri CJ, Nathan SD, Barnett SD, et al. Prevalence and outcomes of pulmonary arterial hypertension in advanced idiopathic pulmonary fibrosis. Chest. 2006;129:746-52.

11. Global Strategy for the Diagnosis, Management and Prevention of COPD, Global Initiative for Chronic Obstructive Lung Disease (GOLD). Publication list; 2017. http://goldcopd.org/gold-2017-global-strategy-diagnosismanagement-prevention-copd/.

12. Ruiter $\mathrm{G}$, Lankhorst $\mathrm{S}$, Boonstra $\mathrm{A}$, et al. Iron deficiency is common in idiopathic pulmonary arterial hypertension. Eur Respir J. 2011;37:1386-91.

13. Ruiter $\mathrm{G}$, Lanser IJ, de Man FS, et al. Iron deficiency in systemic sclerosis patients with and without pulmonary hypertension. Rheumatology (Oxford) 2014:53:285-92.

14. Broberg CS, Bax BE, Okonko DO, et al. Blood viscosity and its relationship to iron deficiency, symptoms, and exercise capacity in adults with cyanotic congenital heart disease. J Am Coll Cardiol. 2006;48:356-65.

15. Rhodes CJ, Howard LS, Busbridge M, et al. Iron deficiency and raised hepcidin in idiopathic pulmonary arterial hypertension clinical prevalence, outcomes, and mechanistic insights. J Am Coll Cardiol. 2011;58:300-9.

16. Van De Bruaene A, Delcroix M, Pasquet A, et al. Iron deficiency is associated with adverse outcome in Eisenmenger patients. Eur Heart J. 2011;32:2790-9.

17. World Health Organization. Iron deficiency anemia. assessment, prevention, and control. A guide for programme managers. Geneva: WHO; 2001.
18. Wedzicha JA, Seemungal TA. COPD exacerbations: defining their cause and prevention. Lancet. 2007:370(9589):786-96.

19. Seemungal TA, Donaldson GC, Paul EA, et al. Effect of exacerbation on quality of life in patients with chronic obstructive pulmonary disease. Am J Respir Crit Care Med. 1998;157(5Pt1):1418-22

20. Pernille A, Petersen T, Pedersen $C T$, et al. Association between hemoglobin and prognosis in patients admitted to hospital for COPD. Int J COPD. 2016;11:2813-20.

21. Yasuda H, Yamaya M, Nakayama K, et al. Increased arterial carboxyhemoglobin concentrations in chronic obstructive pulmonary disease. Am J Respir Crit Care. 2005:171:1246-51.

22. Weiss G, Goodnough LT. Anemia of chronic disease. N Engl J Med. 2005; 352:1011-23.

23. John M, Hoernig S, Doehner W, et al. Anemia and inflammation in COPD. Chest. 2005:127:825-9.

24. Markoulaki D, Kostikas K, Papatheodorou G, et al. Hemoglobin, erythropoietin and systemic inflammation in exacerbations of chronic obstructive pulmonary disease. Eur J Intern Med. 2011;22:103-7.

25. Tanner H, Moschovitis G, Kuster GM, et al. The prevalence of anemia in chronic heart failure. Int J Cardiol. 2002;86:115-21.

26. Cromie N, Lee C, Struthers AD. Anaemia in chronic heart failure: what is its frequency in the UK and its underlying causes? Heart. 2002;87:377-8.

27. Ezekowitz JA, McAlister FA, Armstrong PW. Anemia is common in heart failure and is associated with poor outcomes: insights from a cohort of 12065 patients with new-onset heart failure. Circulation. 2003;107:223-5.

28. Okonko DO, Anker SD. Anemia in chronic heart failure: pathogenetic mechanisms. J Card Fail. 2004;10(Suppl. 1):S5-9.
Ready to submit your research? Choose BMC and benefit from:

- fast, convenient online submission

- thorough peer review by experienced researchers in your field

- rapid publication on acceptance

- support for research data, including large and complex data types

- gold Open Access which fosters wider collaboration and increased citations

- maximum visibility for your research: over $100 \mathrm{M}$ website views per year

At BMC, research is always in progress.

Learn more biomedcentral.com/submissions 